\title{
Primeras citas del género Hypoxylon (Ascomycota, Hypoxylaceae) sobre Ocotea porphyria (Lauraceae)
}

\author{
First Record of the Genus Hypoxylon (Ascomycota, Hypoxylaceae) \\ on Ocotea porphyria (Lauraceae)
}

\author{
Medina, Patricia del V. ${ }^{1 *}$; Esteban B. Sir ${ }^{1-2}$; Adriana I. Hladki ${ }^{1}$ \\ 1 Laboratorio Criptogámico, Fundación Miguel Lillo. Miguel Lillo 251, (4000) San Miguel de Tucumán, \\ Tucumán, Argentina. \\ 2 CONICET. \\ * Autor corresponsal: pmedina@lillo.org.ar
}

\begin{abstract}
- Resumen - Medina, Patricia del V.; Esteban B. Sir; Adriana I. Hladki. 2017. "Primeras citas del género Hypoxylon (Ascomycota, Hypoxylaceae) sobre Ocotea porphyria (Lauraceae)". Lilloa 54 (2). El presente estudio se enmarca en un proyecto cuyo objetivo es conocer la diversidad de ascomicetes asociados al "laurel del cerro". En este primer aporte se estudian las especies de Hypoxylon. Se brinda la primera cita de H. cf. undulatum para la Argentina y se registra a $H$. flavoargillaceum e $H$. lenormandii por primera vez sobre este hospedante. Se incluyen descripciones, ilustraciones y comentarios de las especies.

Palabras clave: Argentina, Laurel, Xylariales.
\end{abstract}

> Abstract - Medina, Patricia del V.; Esteban B. Sir; Adriana I. Hladki. 2017. "First record of the genus Hypoxylon (Ascomycota, Hypoxylaceae) on Ocotea porphyria (Lauraceae)". Lilloa 54 (2). The present study is part of a project whose objective is to know the diversity of ascomicetes associated with "laurel del cerro". In this first contribution we study the species of Hypoxylon. The first record in Argentina of the species H. cf. undulatum is here made, and both $H$. flavoargillaceum and $H$. lenormandii, are registered for the first time on this host. Descriptions, illustrations and comments of the species are included.

Keywords: Argentina, Laurel, Xylariales.

\section{INTRODUCCIÓN}

Ocotea porphyria (Griseb.) van der Werff, comúnmente llamada «laurel del cerro» es un árbol de la familia Lauraceae, endémico de Argentina y Bolivia (Fig. 1 B). En nuestro país, esta especie es representativa de la flora del Noroeste Argentino (NOA) encontrándose principalmente en la selva montana entre los 500 a $1500 \mathrm{~m}$ snm (Grau et al., 2010). Son árboles frondosos y de gran porte, que pueden alcanzar 20 o $30 \mathrm{~m}$ de altura y hasta $4 \mathrm{~m}$ de diámetro (Fig.1 A). Son hospedantes de diversas especies de epífitas, lianas y enredaderas, caracterizándose por poseer aceites aromáticos en sus hojas y corteza (Digilio y Legname, 1966; Meyer, 1963).

Recibido: 22/06/17 - Aceptado: 12/10/17
Debido a que es escaso el conocimiento de la micobiota de ascomicetes asociados al «laurel del cerro», surgió la necesidad de investigar la diversidad fúngica presente en su madera, corteza, hojas y frutos, con el objetivo de identificar, caracterizar y preservar estos microorganismos.

Los reportes de hongos xilariales asociados a este árbol, se restringen a las investigaciones de Sir et al. (2012) quienes citaron a Daldinia eschscholtzii (Ehrenb.) Rehm., Kretzschmaria clavus (Fr.) Sacc., Xylaria aff. ianthinovelutina (Mont.) Fr., X. curta Fr., $X$. fissilis Ces., $X$. globosa (Pers.) Mont., $X$. melanura (Lév.) Sacc., X. mellissi (Berk.) Cooke y X. telfairii (Berk.) Sacc., en sus relevamientos de la micobiota presente en las yungas del país. 
En esta primera contribución nos centraremos en el estudio de las especies de Hypoxylon Bull. halladas en corteza y madera de laurel, considerando que no existen registros previos para el hospedante investigado.

El género Hypoxylon cuenta con más de 150 especies, constituyendo uno de los grupos más diversos y complejos dentro de las Xylariales (Miller, 1961; Ju y Rogers, 1996). Fue erigido por Bulliard en 1791 y desde entonces el concepto genérico se fue reduciendo acompañado por la segregación de muchos géneros a partir del mismo (Hseih et al., 2005; Kuhnert et al., 2015; Fournier et al., 2015). Recientemente algunos estudios de filogenia molecular y una visión polifásica taxonómica de géneros hipoxiloides, permitió proponer una nueva familia denominada Hypoxylaceae (Wendt et al., 2017).

Los primeros registros del género Hypoxylon para la Argentina, fueron realizados por Spegazzini a fines del siglo XIX (1880, 1881, 1887, 1888, 1899, 1908, $1909,1919)$. Posteriormente Hladki y Romero (2006, 2009a, 2009b) efectuaron una actualización taxonómica y nomenclatural de las especies propuestas por Spegazzini, aportando además, nuevas colecciones e incrementando el conocimiento de la diversidad en el país.

Recientemente nuevos estudios acerca de los hongos xilariáceos en el NOA revelaron la presencia de 20 especies de Hypoxylon en las selvas de montaña de Argentina (Sir et al., 2016).

Con el objetivo de ampliar el conocimiento sobre la micobiota fúngica en bosques de laurel del cerro, se presentan tres especies de Hypoxylon creciendo sobre este hospedante.

\section{MATERIALES Y MÉTODOS}

Los ejemplares fueron coleccionados durante exploraciones fúngicas llevadas a cabo en la Reserva Provincial de Flora y Fauna Acambuco -R.P.F.F. Acambuco-, provincia de Salta, Argentina, durante otoño de 2015 (Fig. 1C). También se estudiaron colecciones depositadas en el Herbario Criptogámico LIL de la Fundación Miguel Lillo. Las preparaciones, observaciones microscópicas, y la terminología empleada para las descripciones, se realizaron siguiendo a Ju y Rogers (1996). Para la determinación de los colores en las descripciones de las especies se tuvo en cuenta la carta de colores de Rayner (1970). Para la validación de la posición taxonómica del hospedante en estudio, se consultó la base de datos del Missouri Botanical Garden (http://www.tropicos.org/).

\section{RESULTADOS}

Se identifican tres especies de Hypoxylon (Ascomycota, Hypoxylaceae) creciendo sobre corteza y madera de Ocotea porphyria.

Hypoxylon flavoargillaceum J. H. Mill., en Chardón \& Toro, Monografía de la Universidad de Puerto Rico, Series B 2: 200 (1934).

Figs. 2 A-D y 3 A-B

Teleomorfo.- Estroma glomerado a efuso, con contornos periteciales conspicuos, gránulos castaños amarillentos entre peritecios; pigmentos castaño rojizos (dark brick60) liberados en $\mathrm{KOH}$ al $10 \%$. Peritecios esféricos 0,150,16 x 0,150,2 mm, con ostíolos umbilicados, rodeados por un disco elevado. Ascos 8-esporados, cilíndricos, 74,8-243,1 x 4-8 $\mu \mathrm{m}$, región fértil 39,5-119 $\mu \mathrm{m}$, aparato apical I+, con forma de disco, 2,2-2,8 x 0,3-0,7 $\mu \mathrm{m}$. Ascosporas castaño oscuras, unicelulares, inequiláteras, con extremos redondeados estrechos, 14,9-17 x 7,6-9,1 $\mu \mathrm{m}$; surco germinativo recto, de igual longitud que la espora, en la cara convexa. Perisporio dehiscente en $\mathrm{KOH}$ al 10\%, ornamentado. Episporio liso.

Material estudiado.-ARGENTINA. Prov. Salta, Dpto. Gral. José de San Martín. R.P.F.F. Acambuco, $22^{\circ} 20^{\prime}$ 40,5"S 63 50' 01,3"O, $804 \mathrm{~m}$ snm, madera de Ocotea porphyria, 21V-2015; Medina y Hladki 05 (LIL 162000).

Materiales adicionales estudiados.- ARGENTINA. Prov. Jujuy, Dpto. Santa Bárba- 

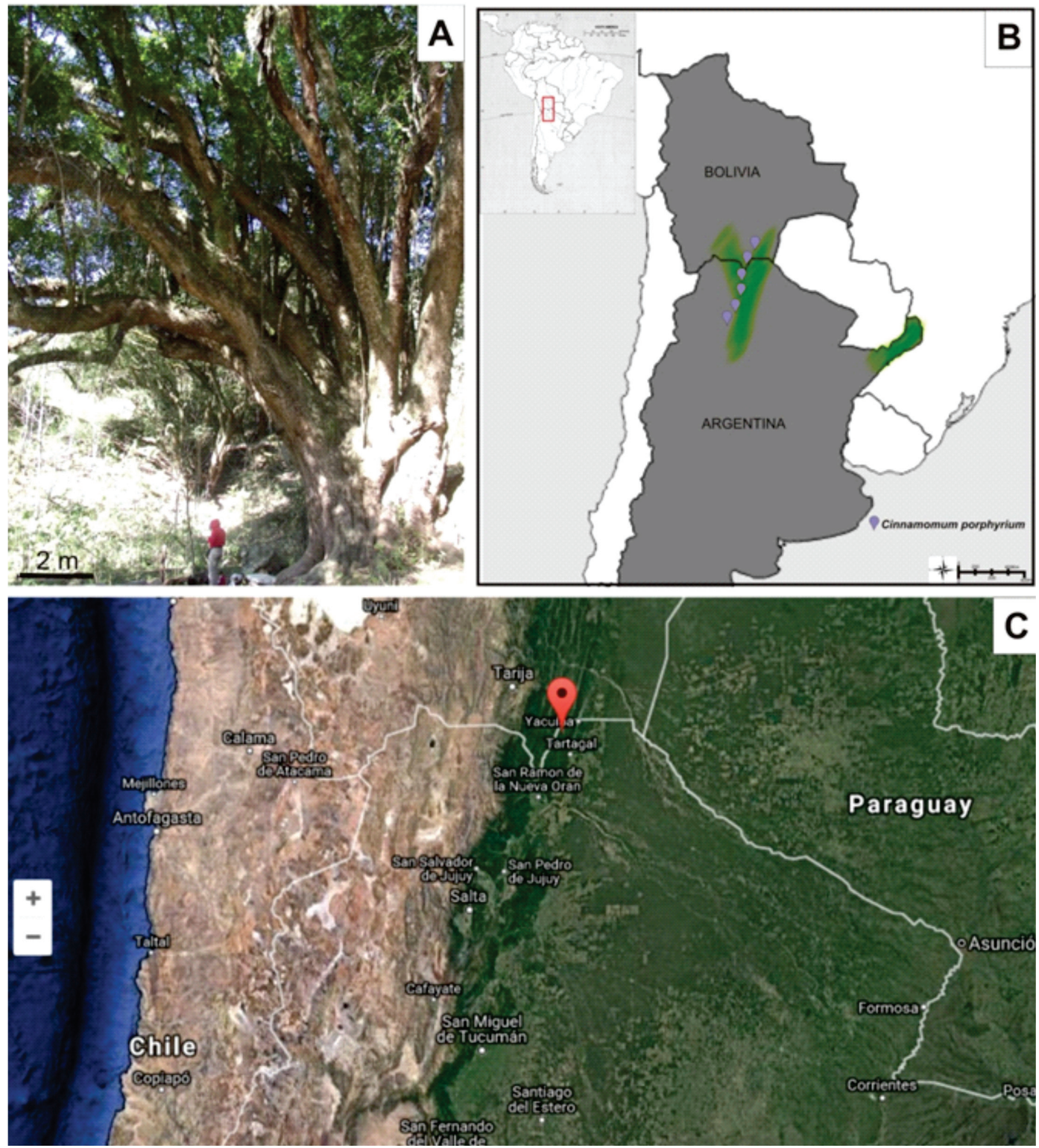

Fig. 1. Ocotea porphyria. A) Aspecto general del hospedante. B) Área de distribución. C) Sitio de muestreo R.P.F.F. Acambuco (Salta, Argentina). Imagen de satélite digital Landsat 6 Google Earth (http://earth.google.com).

ra, Reserva Provincial Las Lancitas, corteza de una rama muerta de Celtis tala Gillies ex Planch (Cannabaceae), asociado con $H$. umbilicatum Speg., 27-IV-2014, Sir y Hladki 679, 687 (LIL). Prov. Salta, Dpto. Anta, P.N. El Rey, corteza de una rama seca de Celtis tala, 14-V-2012, Sir y Hladki 208 (LIL); Dpto. Gral. José de San Martín, R.P.F.F. Acambuco, corteza de una rama seca de Celtis tala, 22IV-2014, Sir y Hladki 503 (LIL).

Distribución geográfica.- La especie es citada sólo para el Neotrópico; Colombia, Venezuela (Ju y Rogers, 1996) y Argentina (Sir, 2016). 
A
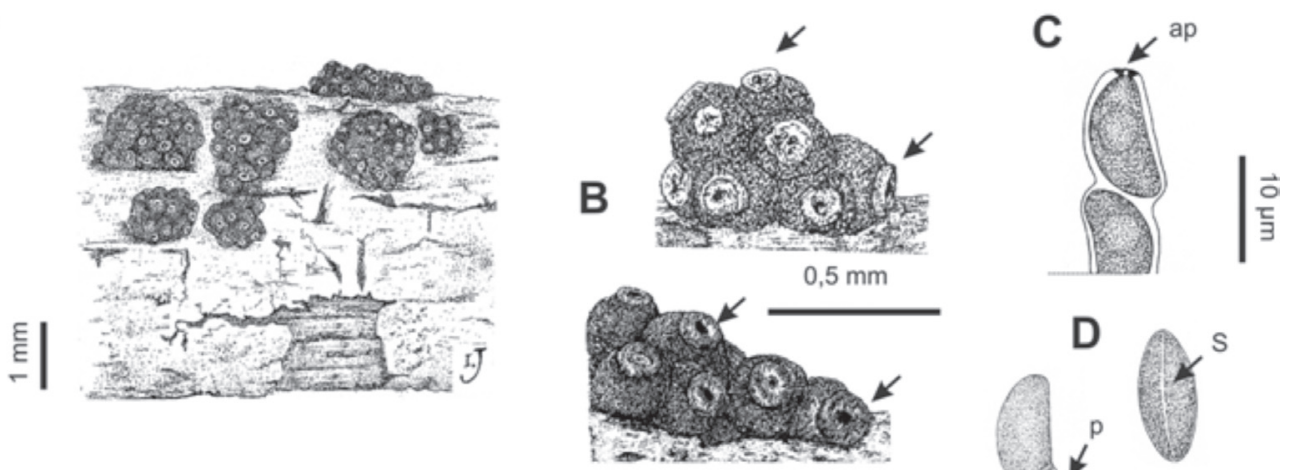

E
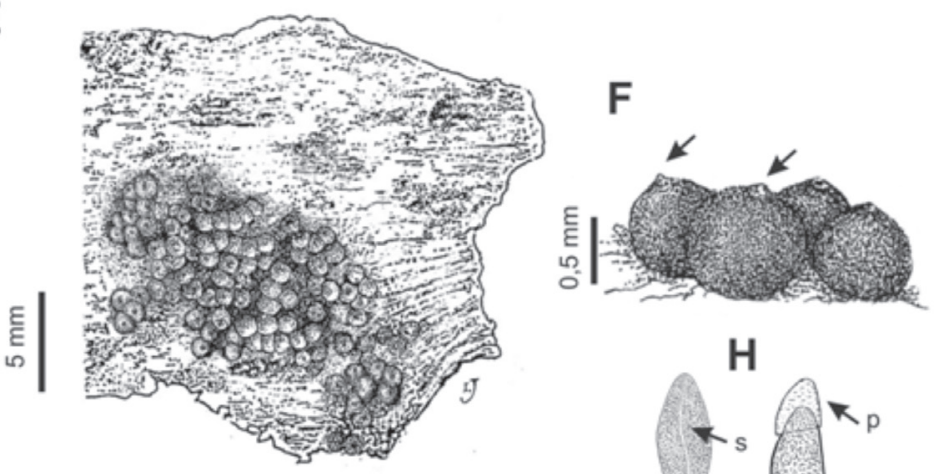

G

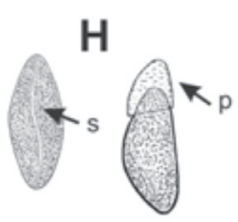

I
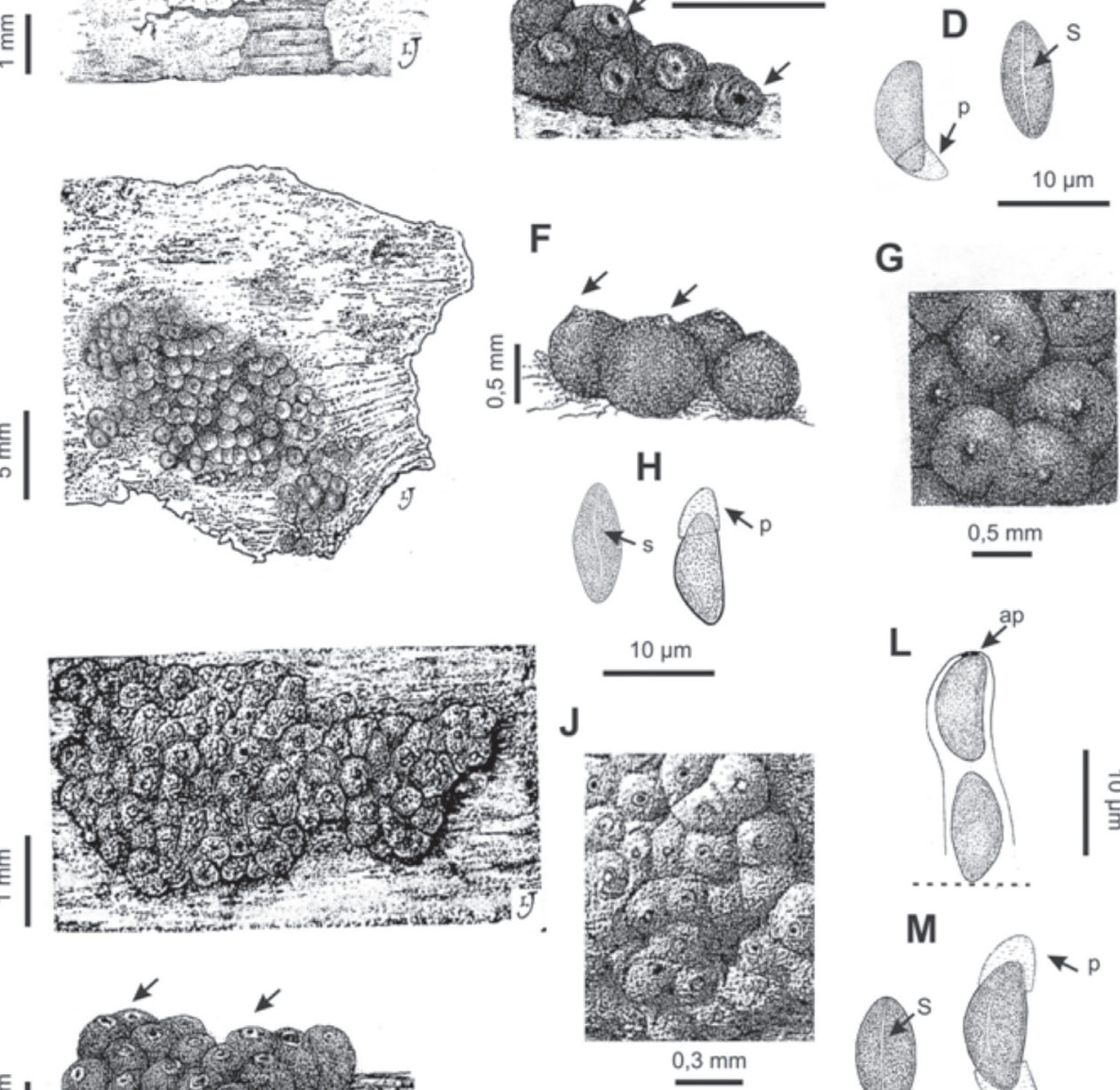

$0,5 \mathrm{~mm}$
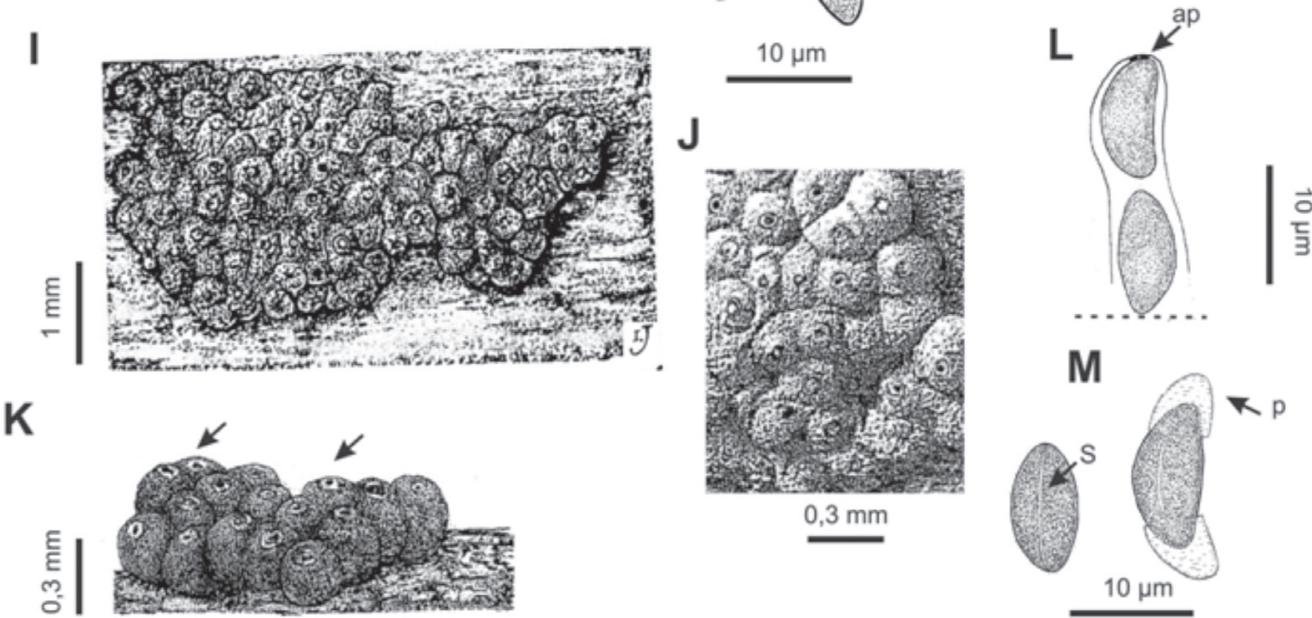

Fig. 2. Hypoxylon flavoargillaceum. A) Aspecto general del estroma glomerado a efuso sobre el sustrato. B] Detalle el estroma; ostíolos umbilicados rodeados de un tejido estromático con forma de disco [flechas]. C) Porción apical de un asco mostrando el aparato apical Iz . D) Ascospora mostrando perisporio dehiscente con ornamentaciones y surco germinativo recto. Medina y Hladki 05 (LIL 162000). Hypoxylon lenormandii. E) Aspecto general del estroma peritecioide. F) Detalle de las papilas ostiolares cónicas (flechas). G) Detalle de los contornos periteciales evidentes. H] Ascosporas mostrando surco germinativo sigmoide y perisporio dehiscente, levemente ornamentado. Medina y Hladki 15 (LIL 162001). Hypoxylon cf. undulatum. I] Aspectos general del estroma efuso pulvinado. J) Vista superficial del estroma. K] Vista lateral mostrando los ostíolos levemente papilados (flechas). L) Porción apical de un asco mostrando el aparato apical con forma de disco lz. M) Ascosporas mostrando surco germinativo recto y perisporio dehiscente, levemente ornamentado. Medina y Hladki 48 (LIL 162002). Referencias: Ap, aparato apical; P, perisporio; S, surco germinativo. 
Observaciones.- Hypoxylon flavoargillaceum se distingue principalmente de $H$. notatum Berk. \& M.A. Curtis, especie muy similar, también presente en las Yungas de Argentina, por tener aparato apical amiloide.

Esta especie fue reportada por Sir (2016) creciendo sobre Citrus L. (Rutaceae) y Celtis tala en las provincias de Salta y Jujuy (Argentina), y en esta oportunidad se cita por primera vez sobre un hospedante de la familia Lauraceae.

Hypoxylon lenormandii Berk. \& M. A. Curtis [como «lenormandi»], en Berkeley, J. Linn. Soc., Bot. 10 ( $\mathrm{N}^{\circ}$ 46): 385 (1868) [1869]

Figs. 2 E-H y 3 C-D

Descripción de teleomorfo y anamorfo.Ver Kuhnert et al. (2015).

Material estudiado.- ARGENTINA. Prov. Salta, Dpto. Gral. José de San Martín. R.P.F.F. Acambuco, $22^{\circ} 16^{\prime} 43,9 " S$ 63 50' 43,3"O, 865 m snm; madera de Ocotea porphyria, 21V-2015; Medina y Hladki 15 (LIL 162001).

Materiales adicionales estudiados.- ARGENTINA. Prov. Jujuy, Dpto. Ledesma, P.N. Calilegua, 11-V-2012, Sir y Hladki 017 (LIL). Prov. Salta, Dpto. Gral. José de San Martín, R.P.F.F. Acambuco, 22-IV-2014, Sir y Hladki 522 (LIL). Prov. Tucumán, Dpto. Capital, jardín de la Fundación Miguel Lillo, 08-X-2007, Hladki 4011 (LIL).

Distribución geográfica.- Pantropical (Ju y Rogers, 1996; Fournier et al., 2015).

Observaciones.- Hypoxylon lenormandii se diferencia de las otras dos especies citadas en este trabajo por presentar estroma efuso-pulvinado con tendencia a ser peritecioide, pigmentos castaño oscuros liberados con OHK, papila ostiolar cónica y ascosporas con surco germinativo ligeramente sigmoide en cara convexa.

Es una especie muy común en los ambientes tropicales y subtropicales; se la en- cuentra creciendo sobre monocotiledóneas y dicotiledóneas (Ju y Rogers, 1996.), sin embargo, poco se conoce sobre la identidad específica de sus hospedantes. En Tailandia se la registró en Albizia chinensis (Osbeck) Merr. (Fabaceae), mientras que en las selvas del NOA es, frecuentemente hallada sobre Tipuana tipu (Benth.) Kuntze (Fabaceae) (Kuhnert et al., 2015).

Se cita por primera vez sobre un hospedante de la familia Lauraceae.

Hypoxylon cf. undulatum Y. M. Ju, J. D. Rogers \& Laessøe, en Ju \& Rogers, Mycol. Mem. 20: 199 (1996). Figs. 2 I-M y 3 E-F

Teleomorfo. - Estroma efuso a pulvinado, superficie castaño rojizo oscuro (brown vinaceous-84), gránulos castaños alrededor de los peritecios, pigmentos amarillos anaranjados (cinnamom-62) liberados en $\mathrm{KOH}$ al 10\%. Peritecios esféricos 0,1-0,2 x 0,1-0,2 $\mathrm{mm}$; ostíolos levemente papilados. Ascos no persistentes, aparato apical I+, con forma de disco, 1,6-2,3 x 0,6-0,9 $\mu \mathrm{m}$. Ascosporas castañas a castañas oscuras, unicelulares, elipsoidales, inequiláteras, 9,8-11,7 x 4,2-5,9 $\mu \mathrm{m}$; surco germinativo recto en cara convexa, de igual longitud que la espora. Perisporio dehiscente en $\mathrm{KOH}$ al 10\%, levemente ornamentado. Episporio liso.

Material estudiado.- ARGENTINA. Prov. Salta, Dpto. Gral. José de San Martín. R.P.F.F. Acambuco, $22^{\circ} 05^{\prime}$ 55,6" S $63^{\circ}$ 56' 31,0" O, $948 \mathrm{~m}$ snm, madera de Ocotea porphyria, 22V-2015; Medina y Hladki 48 (LIL 162002).

Distribución geográfica.- Islas Vírgenes (USA) (Ju y Rogers, 1996).

Observaciones. - El ejemplar aquí presentado exhibe caracteres morfológicos similares a $H$. undulatum; sin embargo en solución de $\mathrm{KOH}$ éste liberó pigmentos que no fueron hallados en el espécimen tipo procedente de las Islas Vírgenes (USA). Los pigmentos son un carácter diagnóstico de gran importancia para la identificación de las especies de 
este género (Ju y Rogers, 1996). Además, se observó que el ancho del aparato apical es menor al que presenta el especimen Tipo
(0,6-0,9 vs 1,5-2 $\mu \mathrm{m})$. Sin embargo se necesitaría contar con nuevas colecciones que permitan encarar estudios complementarios
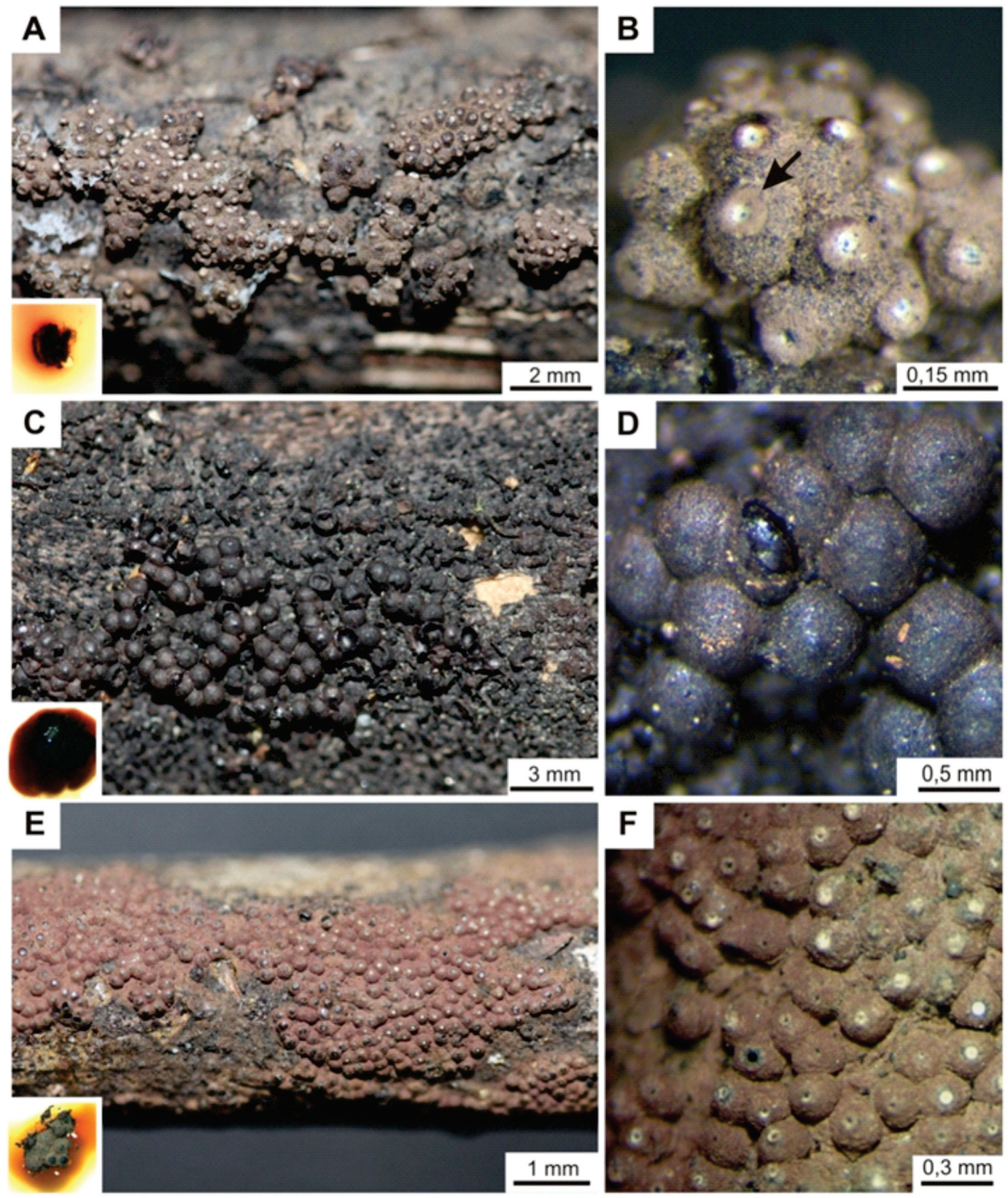

Fig. 3. Hypoxylon flavoargillaceum. A] Aspecto general del estroma y pigmentos castaño rojizos liberados en $\mathrm{KOH}$. B] Detalle de los contornos periteciales y los ostíolos rodeados de un tejido estromático con forma de disco (flecha). Medina y Hladki 05 (LIL 162000). Hypoxylon lenormandii. C) Aspecto general del estroma y pigmentos castaño oscuro liberados en $\mathrm{KOH}$. D) Detalle del estroma peritecioide. Medina y Hladki 15 (LIL 162001). Hypoxylon cf. undulatum. E) Aspecto general del estroma y pigmentos amarillo anaranjados liberados en $\mathrm{KOH}$. F] Detalle del estroma peritecioide. Medina y Hladki 48 (LIL 162002). 
(moleculares y químicos) para poder establecer una correcta caracterización de los especímenes argentinos. Por este motivo, se incluye de forma preliminar a $H$. cf. undulatum como parte de la micobiota asociada al laurel del cerro.

\section{CONCLUSIÓN Y DISCUSIÓN}

Los bosques tropicales son los que presentan mayor diversidad biológica del mundo (Medrano, 2002) y en nuestro país, «Las Yungas» concentran alrededor del 50\% de la biodiversidad de Argentina (Cabrera, 1971; Cabrera y Willink, 1980).

Poco se conoce sobre la relación entre la micobiota xilarial y las distintas especies arbóreas nativas de las Yungas (Sir et al., 2012; Catania y Romero, 2010, 2014). Un estudio sobre la micobiota asociada a bosques de Podocarpus parlatorei Pilg. fue encarado por Catania (2009), identificando 92 especies de ascomicetes, de los cuales 16 especies (17\%) son Xylariales.

Por lo general el sustrato se encuentra en un estado de descomposición muy avanzado cuando se desarrollan los estromas de Hypoxylon, lo que hace dificultosa la identificación taxonómica del árbol; sin embargo la especificidad del hospedante-hongo es un dato valioso, teniendo en cuenta que gran cantidad de especies de Hypoxylon son endófitos.

Por lo tanto, este estudio es el primer resultado de un proyecto amplio, cuyo objetivo es identificar y caracterizar los hongos que habitan sobre O. porphyria en las Yungas argentinas. En esta contribución se dan a conocer la presencia de tres especies de Hypoxylon ( $H$. flavoargillaceum, $H$. lenormandii, $H$. cf. undulatum), registradas por primera vez sobre este hospedante.

\section{BIBLIOGRAFÍA}

Bulliard P. 1791. Histoire des champignons de la France. I. Editorial Leblanc, Paris, $368 \mathrm{pp}$.

Cabrera A. 1971. Fitogeografía de la República Argentina. Boletín de la Sociedad Argentina de Botánica 14 (1-2): 1-43.

Cabrera A., Willink A. 1980. Biogeografía de América Latina. $2^{a}$ edición corregida. Serie de Biología. Monografía 13. Editorial Secretaría General de la Organización de los Estados Americanos, Washington D. C.,122 pp.

Catania M. 2009. Diversidad de especies de Hymenoascomycetes y Loculoascomycetes sobre Podocarpus parlatorei Pilg., en las provincias de Tucumán y Catamarca. Tesis doctoral. Universidad Nacional de Tucumán, Argentina, 386 pp.

Catania M., Romero A. I. 2010. Micromicetes asociados a la corteza y madera de Podocarpus parlatorei (Podocarpaceae) en la Argentina. VI. Ascomycota. Darwiniana 48 (2): 123-140.

Catania M., Romero A. I. 2014. Micromicetes asociados a corteza y madera de Podocarpus parlatorei (Podocarpaceae) en la Argentina. VIII. Rosellinia (Xylariaceae, Ascomycota). Darwiniana, nueva serie 2 (1): 57-67.

Digilio A., Legname P. 1966. Los árboles indígenas de la provincia de Tucumán. Opera Lilloana 15: 1-107.

Fournier J., Lechat C., Courtecuisse R. 2015. The genus Hypoxylon (Xylariaceae) in Guadeloupe and Martinique [French West Indies.). Ascomycete.org 7 (5): 145-212.

Grau H. R., Paolini L., Malizia A., Carilla J. 2010. Distribución, estructura y dinámica de los bosques de las Sierras de San Javier, Tucumán. En: Grau H. R. (editor), Ecología de una interfase naturalurbana. La Sierra de San Javier y el Gran San Miguel de Tucumán. Editorial de la U.N.T., Tucumán, 237 pp.

Hladki A. I., Romero A. I. 2006. Revisión de las especies de Hypoxylon propuestas por Spegazzini. Lilloa 43 (1-2): 45-60.

Hladki A. I., Romero A. I. 2009a. Taxonomic and nomenclatural aspects of Hypoxylon taxa from Southern South America proposed by Spegazzini. Mycología 101 (3): 733-744.

Hladki A. I., Romero A. I. 2009b. Novedades para los géneros Annuhypoxylon e Hypoxylon (Ascomycota-Xylariaceae) en la República Argentina. Darwiniana 47 (2): 278-288.

Hsieh M., Ju Y. M., Rogers J. 2005. Molecular phylogeny of Hypoxylon and closely related genera. Mycología 97: 844865.

Ju Y. M., Rogers J. 1996. A revision of the genus Hypoxylon. Mycologia Memoir $\mathrm{N} \square$ 20. Editorial The American Phytopathological Society (APS) Press, St. Paul, Minnesota. 199pp. 
Kuhnert E., Surup F., Sir E. B., Lambert C., Hyde K. D., Hladki A. I., Romero A. I., Stadler M. 2015. Lenormandins A-G, new azaphilones from Hypoxylon lenormandii and Hypoxylon jaklitschii sp. nov., recognized by chemotaxonomic data. Fungal Diversity 71: 165-184.

Medrano G. 2002. Biodiversidad, un recurso no valorado. (http://www. ecoportal.net/ Temas-Especiales/Biodiversidad/BIODIVERSIDAD_-_Un_Recurso_No_Valorado]

Meyer T. 1963. Estudio sobre la Selva Tucumana. La Selva de Mirtáceas de «Las Pavas». Opera Lilloana 10: 1144.

Miller H. 1961. A monograph of the world species of Hypoxylon. Editorial: University Georgia Press, Athens, 158 pp.

Rayner R. W. 1970. A mycological colour chart. Editorial Commonwealth Mycological Institute, Kew and British Mycological Society, Great Britain. 34 pp

Sir E. B. 2016. Biodiversidad de la familia Xylariaceae (Ascomycota) en el centronorte de las Yungas de Argentina. Tesis Doctoral. Universidad Nacional de Tucumán, Argentina, 413 pp.

Sir E. B., Hladki A. I., Parrado M. F., Romero A. I. 2012. Biodiversity of Xylariaceae (Ascomycota) and their hosts in protected areas from Tucumán (Argentina). Kurtziana 37 (2): 35-48.

Sir E. B., Kuhnert E., Lambert C., Hladki A. I., Romero A. I., Stadler M. 2016. New species and reports of Hypoxylon from Argentina recognized by a polyphasic approach. Mycology Progress 15: 142.
Spegazzini C. L. 1880. Fungi Argentini. Anales de la Sociedad Científica Argentina 9 (4): 179-180.

Spegazzini C. L. 1881. Fungi Argentini additis nonnullis brasilensibus montevideensibusque. Anales de la Sociedad Científica Argentina 12 (3): 108-109.

Spegazzini C. L. 1887. Fungi Patagonici. Boletín de la Academia Nacional de Ciencias de Córdoba 11(1): 5-64.

Spegazzini C. L. 1888. Fungi Fuegiani. Boletín de la Academia Nacional de Ciencias de Córdoba 11 (2): 200-204.

Spegazzini C. L. 1899. Fungi Argentini novi vel critici. Anales del Museo Nacional de Historia Natural de Buenos Aires 6: 264-265.

Spegazzini C. L. 1908. Hongos de la yerba mate. Anales del Museo Nacional de Historia Natural de Buenos Aires 17 (10): 111-141.

Spegazzini C. 1909. Mycetes Argentinenses. Anales del Museo Nacional de Historia Natural de Buenos Aires 19 (12): 343344.

Spegazzini C. L. 1919. Los hongos de Tucumán. Primera Reunión Nacional de la Sociedad Argentina de Ciencias Naturales de Tucumán. $267 \mathrm{pp}$

Wendt L., Sir E. B., Kuhnert E., Heitkämper S., Lambert C., Hladki A. I., Romero A. I., Luangsa-ard J. J., Srikitikulchai P., Persoh D., Stadler M. 2017. Resurrection and emendation of the Hypoxylaceae, recognized from a multigene phylogeny of the Xylariales. Mycology Progress. DOl 10.1007/s 11557-0171303-3. 